López-Chao, V., Mato-Vázquez, D., y Chao-Fernández, R. (2020). Análisis confirmatorio de la estructura factorial de la ansiedad hacia las matemáticas. Revista de Investigación Educativa, 38(1), 221-237.

DOI: http://dx.doi.org/10.6018/rie.359991

\title{
Análisis confirmatorio de la estructura factorial de la ansiedad hacia las matemáticas
}

\author{
Confirmatory factor analysis of mathematics anxiety \\ Vicente López-Chao*, Dorinda Mato-Vázquez** y Rocío Chao-Fernández** \\ * Universidad de la Laguna \\ **Universidade da Coruña
}

\begin{abstract}
Resumen
Esta investigación replantea la estructura factorial de la ansiedad hacia las matemáticas propuesta por Mato-Vázquez (2006), a través del estudio de una muestra de 1220 estudiantes de Educación Secundaria Obligatoria. El modelo de ecuaciones estructurales sugiere un modelo basado en cuatro escalas: "Ansiedad hacia la demostración del conocimiento matemático", "Preocupación por la presencia inevitable de las matemáticas", "Ansiedad hacia la acción matemática" y "Ansiedad hacia la actividad matemática en el entorno No formal". Posteriormente, se ha analizado mediante análisis de regresión lineal múltiple (paso a paso), con el objetivo de predecir el rendimiento académico en matemáticas medido a través de la calificación del curso anterior. Los resultados muestran un mayor porcentaje de predicción en el curso más alto, lo que confirma la importancia de la ansiedad como variable predictora en el rendimiento $y$, por lo tanto, su influencia determinante en el proceso de enseñanza y aprendizaje, así como los resultados académicos adquiridos.

Palabras clave: matemáticas; ansiedad; rendimiento; alumnado.
\end{abstract}

\begin{abstract}
This paper rethinks the factorial structure of mathematics anxiety by Mato-Vázquez (2006), through the study of a sample of 1220 students of Compulsory Secondary Education. The structural equations' model suggests a four-scale format comprised of "Anxiety about

Correspondencia: Vicente López-Chao, vlopezch@ull.edu.es. Escuela Politécnica Superior de Ingeniería. Avenida Ángel Guimerá Jorge, s/n. 38200. San Cristóbal de La Laguna. S/C de Tenerife (España).
\end{abstract}


demonstrating mathematical knowledge", "Concern over mathematics' presence", "Anxiety about mathematical action" and "Anxiety about mathematical activity in non-formal contexts". It has been examined through a multiple linear regression analysis (conducted step by step), with the aim of predicting the students' academic performance in Mathematics courses, which has been measured by previous course grades. Results show a higher prediction percentage in the fourth year, confirming the extent to which anxiety as a predictor variable influences the students' performance and, therefore, its determining impact on the teaching and learning process, as well as on the academic results acquired.

Keywords: mathematics; anxiety; academic performance; students.

\section{Introducción}

El número de trabajos que profundizan en la enseñanza y aprendizaje de las Matemáticas se ha incrementado en los últimos años. La razón principal la auguraba ya la National Council of Teachers of Mathematics (NCTM) en el año 2003 al afirmar que la demanda de una buena preparación en esta área de conocimiento seguiría aumentando cada vez más, ya que saber Matemáticas es una necesidad en cualquier sociedad y, actualmente, se hacen inevitables en todos los ámbitos de la vida diaria.

Sorprende pues que los resultados académicos y las evaluaciones externas del Ministerio de Educación, Cultura y Deporte (MECD, 2012) y la Organización para la Cooperación y el Desarrollo Económicos (OCDE, 2012) muestren a los estudiantes españoles con una preparación deficiente en Matemáticas. Sin entrar a analizar el foco controvertible en el que se centran estos estudios, la realidad es que la puntuación de nuestro alumnado es inferior a la mayoría de los países de nuestro entorno.

Los motivos para tal escenario son varios, y aparte de que el alumnado no sabe utilizar lo aprendido en situaciones usuales de la vida cotidiana y las competencias en Matemáticas no están siendo parte esencial de esa preparación, parece evidente que existen muchos escolares, independientemente de la edad, curso, ámbitos, tipos de centro, nivel socioeconómico y familiar con sentimientos negativos hacia las Matemáticas (Mato-Vázquez, 2014).

A nivel general, Palacios, Arias y Arias (2014) y Caballero, Guerrero y Blanco (2014), entre otros, afirman que es de las asignaturas más odiadas del currículo, y la que produce en los escolares más desinterés, insatisfacción por el trabajo, falta de autoestima, insuficiente confianza en las propias destrezas, actitudes negativas hacia la escuela y escaso rendimiento matemático y científico. Y es que, los sentimientos negativos, casi siempre, van a la par de las bajas calificaciones; de la misma manera que los positivos influyen en el agrado y en un mayor éxito en la asignatura (Carrell, Page y West, 2010).

En esa línea, Gómez-Chacón (2010) corrobora que un buen número de estudiantes está convencido de que la asignatura "es difícil", "no sirve para nada", "no la entienden", y otras opiniones generalizadas similares que se escuchan a diario en los centros educativos. Es tal el malestar que produce en algunas personas que pueden llegar a sentir angustia, náuseas, nervios y ansiedad durante toda su vida (Ashcraft, 2002; Hannula, 2012).

Uno de los principales estudiosos de la ansiedad hacia las matemáticas es McLeod (1992), quién entiende este constructo como un estado de ansiedad en respuesta a las 
situaciones relacionadas con las Matemáticas, que se aprecia como una amenaza hacia la propia autoestima de la persona que la padece al enfrentarse a problemas matemáticos, operaciones numéricas o aritmética.

Desde la perspectiva de Yara (2009) y Khatoon y Mahmood (2010) las matemáticas suponen estrés, tensión y esfuerzo en el cuerpo y en la mente tanto en su vida cotidiana como académica, por lo que muchos alumnos se sienten enfermos cuando intentan hacer algo relacionado con ellas; empiezan a sudar y a temblar como reacción a la humillación pública, se sienten desesperados si no saben muy bien los temas o dejan algo sin terminar, y les hace sudar las manos, les revuelve el estómago y les produce dolor de cabeza.

Tal como ponen de manifiesto Casis, Rico y Castro (2017), la ansiedad hacia las Matemáticas puede tomar formas multidimensionales incluyendo, por ejemplo, aversión (un elemento de actitud), preocupación (un elemento cognitivo) y miedo (un elemento emocional), o puede ser un reflejo de otras actitudes más profundamente asentadas hacia las Matemáticas.

Por otra parte, el énfasis está en que altos niveles de ansiedad influye en el proceso cognitivo de las personas: la atención y la memoria se predisponen hacia la información que van a recibir, y la realización de las tareas cognitivas se ve alterada (Hannula, 2012); reducen, por lo tanto, la eficiencia en el aprendizaje, pues disminuyen la concentración con el consecuente deterioro en el rendimiento escolar (Delgado, Inglés y García-Fernández, 2013). También interfiere en los procesos de pensamiento (Khezri, Lavasania, Malahmadia y Amania, 2010), disminuye la disciplina (Iriarte y Benavides, 2011), e impide que el individuo sea consciente del potencial que tiene (Belbase, 2013).

En muchas ocasiones, la ansiedad hacia las Matemáticas es un factor no intelectual en el sentido de que fue observado en estudiantes con gran capacidad, competentes y con un alto rendimiento en otras materias (Blanco, Guerrero y Caballero, 2013). Por lo que muchos alumnos prescinden de las carreras científicas que tengan esta asignatura (Galla y Wood, 2012); lo que supone serias consecuencias para las opciones educativas y elecciones profesionales, además de sentimientos negativos de culpa y vergüenza (Pérez-Tyteca, Monje y Castro, 2013). Es decir, condiciona su futuro (Bekdemir, 2010).

Entre los precursores se encuentran Richardson y Suinn (1972), que describen ciertas variables que pueden afectar a la ansiedad hacia las matemáticas: falta de confianza en las habilidades para resolver problemas difíciles, baja capacidad para aprenderlas, profesores que humillan a los alumnos, metodologías de enseñanza en las que solo hay una forma de resolver un problema, una única respuesta correcta, un profesor autoritario (Gómez-Chacón, 2009 en Gamboa Araya y Moreira-Mora, 2016), la cercanía para realizar un examen (Asikhia y Mohangi, 2015).

Los exámenes son, junto con las técnicas de estudio y los problemas emocionales, la causa más habitual de ansiedad para la mayoría de los escolares (Bekdemir, 2010). Muchos sujetos sufren angustia aguda antes y durante los exámenes, que se incrementa de manera significativa con la edad.

En términos generales, parece que muchos estudiantes se ponen nerviosos y experimentan de manera característica una gran tensión cuando se les llama para contestar preguntas, bien sea en público o ante las evaluaciones (Eden, Heine y Jacobs, 2013). Esto es debido, entre otros motivos a la falta de autoestima y vergüenza a la hora de reconocer que no se entiende un concepto (Hadfield y McNeil en Bekdemir, 2010). 
En cualquier caso, se sabe que los alumnos van tomando conciencia de sus capacidades, motivación o frustración hacia las Matemáticas desde los primeros años, y que la ansiedad es mayor conformen avanzan los cursos (Broc Cavero, 2006). En esta misma línea apunta la contribución Ahmed, Minnaert, Kuyper y Werf (2012) quienes señalan que la influencia de experiencias matemáticas negativas en edades tempranas en la escolaridad del alumno es uno de los motivos de ansiedad.

De esta manera, podemos afirmar con investigadores como Gómez-Chacón, Op't Eynde y Corte (2006) que los resultados (calificaciones) no dependen sólo de factores intelectuales, sino que están, también, determinados por las perspectivas y experiencias de los alumnos y por la visión que ellos mismos tienen de sí como estudiantes de matemáticas.

Aunando en lo anterior, resulta pertinente mencionar que el papel que desempeñan los padres, el centro y el curso escolar en la dimensión afectiva de los estudiantes es determinante en el proceso de enseñanza-aprendizaje de las Matemáticas; de hecho, irónicamente, la ansiedad se ve fortalecida en las aulas y en las familias, reforzada por una deficiente habilidad en la realización de actividades matemáticas e inversamente relacionada con actitudes positivas hacia ellas (Blanco, Guerrero y Caballero, 2013).

Por tanto, no deja de ser alarmante, que siendo las Matemáticas una de las asignaturas más importantes del currículo, es de las más temidas en todos los niveles escolares (Frenzel, Pekrun y Goetz, 2007) y la que tiene un rendimiento más deficiente (Bazán y Aparicio, 2006). Y, a medida que se asciende en los cursos escolares, el interés por la asignatura decrece, la utilidad de la materia de cara al futuro sufre un descenso, la competencia percibida para el aprendizaje y logro en las Matemáticas disminuye, surgen los sentimientos y emociones negativas y aumenta la ansiedad. Esto repercute significativamente en la poca implicación y en el menor esfuerzo personal que realiza el estudiante en el proceso de enseñanza-aprendizaje de la asignatura. Como consecuencia, el rendimiento baja y las Matemáticas se convierten en una de las materias más difíciles de enseñar y de aprender (Gresham, 2004; Núñez-Peña, Suárez-Pellicioni y Bono, 2013).

\section{Método}

\section{Objetivos}

Los objetivos de esta investigación se centran en verificar la calidad de la escala "Ansiedad hacia las matemáticas" de Mato-Vázquez (2006) en sus cuatro factores "Ansiedad hacia la demostración de conocimientos matemáticos", "Preocupación ante la presencia inevitable de las matemáticas", "Ansiedad hacia la acción matemática" y "Ansiedad hacia la actividad matemática en entorno no formal", para posteriormente analizar el valor predictivo en el rendimiento académico (última calificación) teniendo en cuenta la variable curso de Educación Secundaria Obligatoria $\left(1^{\circ}, 2^{\circ}, 3^{\circ}\right.$ y $\left.4^{\circ}\right)$.

\section{Población y Muestra}

Los datos para la realización de los análisis cuantitativos se han obtenido a partir de una encuesta realizada entre 1220 alumnos (48\% chicos y 52\% chicas), de cen- 
tros educativos de Educación Secundaria Obligatoria (ESO) (24.1\% primero, 24.5\% segundo, $26.1 \%$ tercero y $25.2 \%$ cuarto curso) en A Coruña (España), mediante un sistema de muestreo no probabilístico casual, lo que se justifica por las facilidades de acceso y la proximidad de los sujetos.

Se ha calculado el error de muestreo (a partir del supuesto de máxima indeterminación ( $\mathrm{p}=\mathrm{q}=.50)$ ) con un margen de confianza de 95\% para una población de 20000 alumnos matriculados en institutos que imparten la ESO en A Coruña, ha sido de 2.7\%, para la muestra conjunta, y de un $4 \%$ para la muestra independiente de hombres y un $3.8 \%$ para la muestra independiente mujeres.

\section{Instrumento}

El instrumento empleado se basó en el diseñado por Mato-Vázquez (2006) con una fiabilidad Alpha de Cronbach de,9504 a partir de la Escala de Ansiedad hacia las Matemáticas (MARS), ideada por Richardson y Suinn en 1972 y aplicado a una muestra de 1.220 alumnos de ESO.

Para nuestro estudio, sometido a la revisión del grupo de expertos, se producen modificaciones y se obtiene un cuestionario de 13 afirmaciones, distribuidas en cuatro subescalas, que analizan la "Ansiedad hacia la demostración de conocimientos matemáticos" (ADCM, 6 ítems), "Preocupación ante la presencia inevitable de las matemáticas" (PPIM, 2 ítems), "Ansiedad hacia la acción matemática" (AAM, 3 ítems) y "Ansiedad hacia la actividad matemática en entorno no formal" (AAMENF, 2 ítems) sobre los cuales los estudiantes han manifestado su grado de acuerdo o desacuerdo siguiendo una escala de tipo Likert, en la que 1 significa "Nada de acuerdo" y 5 "Totalmente de acuerdo".

La subescala ADCM describe la ansiedad que tienen los estudiantes al tener que exponer lo que saben de matemáticas, explicar un problema (ansiedad social), realizar exámenes, esperar las notas finales, ....

La subescala PPIM recoge información acerca del desasosiego que siente el alumnado al no poder escapar de las matemáticas; inquietud y preocupación por no poder.

La subescala AAM hace referencia a la ansiedad que experimenta el estudiante hacia la acción matemática; resolver ejercicios, operaciones matemáticas, manipulación de números y uso de conceptos matemáticos en general...

La subescala AAMENF se refiere a la ansiedad hacia la actividad matemática en entornos no formales; la falta de habilidad para hacer matemáticas por sí mismos fuera del entorno escolar; variedad de situaciones de la vida del individuo en las que puede sentir estrés ante los números (hacer un problema de álgebra, recoger dinero para comprar un ticket ...).

\section{Procedimiento de recogida y análisis de datos}

El procedimiento para la recogida de datos fue de forma presencial en las aulas delante del profesor, y de forma anónima y voluntaria; previamente se explicó a los estudiantes los objetivos de la investigación y se dieron las instrucciones para la adecuada cumplimentación de las escalas. 
Para abordar los objetivos de este estudio, verificar la calidad de la escala y analizar el valor predictivo en el rendimiento académico teniendo en cuenta determinadas variables sociales como el curso académico, se han aplicado dos tipos de técnicas metodológicas. Por un lado, un modelo de ecuaciones estructurales para el análisis de las subescalas utilizadas, lo que nos va a permitir realizar un análisis de corte cuantitativo de acuerdo al modelo teórico propuesto en el cual trabajamos con variables latentes (constructos) que no son susceptibles de medida directa, pero si observadas o ítems de encuestas realizadas (Chin, Peterson y Brown, 2008). Por otro lado, con la certeza de que la ansiedad puede predecir el rendimiento utilizamos, como segunda técnica, un análisis de regresión lineal múltiple para cada una de las categorías de curso.

Las ecuaciones estructurales se perfilan como una técnica adecuada para valorar cuestionarios en el contexto del sistema educativo (González-Montesinos y Backhoff, 2010).

La teoría de la que partimos tiene en cuenta una serie de escalas que miden la ansiedad hacia las matemáticas como hemos puesto de manifiesto anteriormente.

Siguiendo este enfoque se ha construido un modelo conceptual que analiza estos cuatro factores y que en nuestro estudio hemos denominado "ADCM (factor 1), "PPIM" (factor 2), "AAM" (factor 3), y "AAMENF" (factor 4).

De acuerdo con Loehlin, (2004) se ha identificado la composición de las variables latentes, construidas por varias variables observables cada una, que se recogen en la Tabla 1. La "ansiedad del alumnado de ESO hacia las matemáticas" constituye la variable dependiente. Las variables independientes son: (ADCM), (PPIM), (AAM) y (AAMENF).

\section{Tabla 1}

Indicadores reflectivos utilizados para la medición de variables latentes.

\begin{tabular}{lcc}
\hline \multicolumn{1}{c}{ Variables latentes Indicadores reflectivos y descripción } & M & DT \\
\hline Me pongo nervioso cuando abro el libro de matemáticas y encuentro una página llena de problemas & 2.14 & 1.025 \\
Me siento nervioso al pensar en el examen de matemáticas, cuando falta una hora para hacerlo & 2.65 & 1.230 \\
Me siento nervioso cuando escucho cómo otros compañeros resuelven un problema de matemáticas & 3.93 & 1.015 \\
Me pongo nervioso cuando me doy cuenta de que el próximo curso aún tendré clases de matemáticas & 2.53 & 1.184 \\
Me pongo nervioso cuando alguien me mira mientras hago los deberes de matemáticas & 3.76 & 1.044 \\
Me ponen nervioso los exámenes de matemáticas & 3.79 & 1.032 \\
Me pone nervioso hacer operaciones matemáticas & 2.73 & 1.089 \\
Me siento nervioso al tener que explicar un problema de matemáticas al profesor & 3.84 & 1.036 \\
Me siento nervioso cuando me dan una lista de ejercicios de matemáticas & 2.72 & 1.083 \\
Estoy nervioso al recibir las notas finales (del examen) de matemáticas & 3.86 & 1.008 \\
Me siento nervioso cuando quiero averiguar el cambio en la tienda & 1.89 & 1.163 \\
Me siento nervioso cuando tengo que explicar un problema en clase de matemáticas & 3.75 & 1.030 \\
Me siento nervioso cuando empiezo a hacer los deberes & 1.75 & 1.038 \\
\hline
\end{tabular}

Media: M; Desviación típica: DT 


\section{Resultados}

\section{Análisis multivariable. Fiabilidad y validez de las escalas.}

Antes de proceder a la realización del análisis multivariable se analizó la dimensionalidad de los datos obtenidos.

Se realizó la medida de adecuación Muestral Kaiser-Meyer-Olkin (KMO=.841), con un resultado de coeficiente de correlaciones parciales alto, que junto a los valores obtenidos en la prueba de esfericidad de Barlett, con un $\chi_{78}=23966.033(\mathrm{p}<.000)$, permiten rechazar la hipótesis nula de ser una matriz de identidad, lo que indica que existen interrelaciones significativas entre los ítems del instrumento.

A continuación, se ejecutó un análisis de componentes principales con rotación varimax (la rotación ha convergido en siete iteraciones). En la Tabla 2 se presentan los cuatro factores obtenidos que explican el 89,150\% de la varianza, en la que el primer factor (ADCM) explica el 40.177\%, el segundo (PPIM) el 17.574\%, el tercero (AAM) el $16.697 \%$ y el cuarto (AAMENF) el 14.703\%.

Tabla 2

Matriz de componentes rotados.

\begin{tabular}{|c|c|c|c|}
\hline Factor & ítem & n & peso \\
\hline \multirow{6}{*}{$\mathrm{ADCM}$} & $\begin{array}{l}\text { Me pongo nervioso cuando alguien me mira mientras hago los deberes de } \\
\text { matemáticas }\end{array}$ & 6 & .978 \\
\hline & $\begin{array}{l}\text { Me siento nervioso cuando tengo que explicar un problema en clase de } \\
\text { matemáticas }\end{array}$ & 10 & .971 \\
\hline & Me ponen nervioso los exámenes de matemáticas & 5 & .962 \\
\hline & Estoy nervioso al recibir las notas finales (del examen) de matemáticas & 13 & .947 \\
\hline & $\begin{array}{l}\text { Me siento nervioso al tener que explicar un problema de matemáticas al } \\
\text { profesor }\end{array}$ & 12 & .946 \\
\hline & $\begin{array}{l}\text { Me siento nervioso cuando escucho cómo otros compañeros resuelven un } \\
\text { problema de matemáticas }\end{array}$ & 8 & .675 \\
\hline \multirow{2}{*}{ PPIM } & $\begin{array}{l}\text { Me pongo nervioso cuando me doy cuenta de que el próximo curso aún } \\
\text { tendré clases de matemáticas }\end{array}$ & 2 & .920 \\
\hline & $\begin{array}{l}\text { Me siento nervioso al pensar en el examen de matemáticas, cuando falta } \\
\text { una hora para hacerlo }\end{array}$ & 4 & .912 \\
\hline \multirow{3}{*}{ AAM } & $\begin{array}{l}\text { Me pongo nervioso cuando abro el libro de matemáticas y encuentro una } \\
\text { página llena de problemas }\end{array}$ & 11 & .858 \\
\hline & Me pone nervioso hacer operaciones matemáticas & 3 & .804 \\
\hline & Me siento nervioso cuando me dan una lista de ejercicios de matemáticas & 9 & .798 \\
\hline \multirow{2}{*}{ AAMENF } & Me siento nervioso cuando quiero averiguar el cambio en la tienda & 1 & .947 \\
\hline & Me siento nervioso cuando empiezo a hacer los deberes & 7 & .928 \\
\hline
\end{tabular}


La validez de contenido se fundamenta en la coherencia con los estudios y teorías presentes en la literatura.

Al mismo tiempo, se testó la fiabilidad y validez a través del coeficiente Alpha de Cronbach, como vemos en la Tabla 3 con un resultado mínimo de.870 que resulta satisfactorio por ser superior a.70.

Tabla 3

Matriz de componentes rotados.

\begin{tabular}{|c|c|c|c|c|c|}
\hline & & Peso & $\begin{array}{l}\text { Alpha de } \\
\text { Cronbach }\end{array}$ & $\begin{array}{c}\text { Fiabilidad } \\
\text { compuesta } \\
\text { (Composite reliability) }\end{array}$ & $\begin{array}{c}\text { Validez } \\
\text { conve. (AVE) }\end{array}$ \\
\hline \multirow{6}{*}{ ADCM } & ADCM1 & .978 & \multirow{6}{*}{,968 } & \multirow{6}{*}{0,988045936} & \multirow{6}{*}{0.845353167} \\
\hline & ADCM2 & .971 & & & \\
\hline & ADCM3 & .962 & & & \\
\hline & ADCM4 & .947 & & & \\
\hline & ADCM5 & .946 & & & \\
\hline & ADCM6 & .675 & & & \\
\hline \multirow{2}{*}{ PPIM } & PPIM7 & .920 & \multirow{2}{*}{,951 } & \multirow{2}{*}{0,912493475} & \multirow{2}{*}{0.839072} \\
\hline & PPIM8 & .912 & & & \\
\hline \multirow{3}{*}{ AAM } & AAM9 & .858 & \multirow{3}{*}{870} & \multirow{3}{*}{0,749797051} & \multirow{3}{*}{0.673128} \\
\hline & AAM10 & .804 & & & \\
\hline & AAM11 & .798 & & & \\
\hline \multirow{2}{*}{ AAMENF } & AAMENF12 & .947 & \multirow{2}{*}{,928 } & \multirow{2}{*}{0,935595875} & \multirow{2}{*}{0.8789965} \\
\hline & AAMENF13 & .928 & & & \\
\hline
\end{tabular}

Del mismo modo, para complementar este análisis se emplea la fiabilidad compuesta (composity reability) como alternativa que tiene en cuenta las interrelaciones de los constructos extraídos y no se ve influenciada por la cantidad de ítems presentes en la escala. Los resultados obtenidos también son satisfactorios por ser superiores a.5.

En cuanto a la validez de constructo, se utilizó el Análisis Factorial Exploratorio (AFE) por el método de Componentes Principales y rotación Varimax para minimizar el número de variables que tienen saturaciones altas en cada factor y simplificar la interpretación de los factores optimizando la solución por columna. Posteriormente, se utilizó la Varianza Media Extraída (AVE), que al obtener valores superiores a.5 indica que los valores se correlacionan fuertemente y de forma positiva con otras medidas del mismo constructo, siendo en este caso superior para las cuatro dimensiones, con un $84 \%$, un $83 \%$, un $67 \%$ y un $87 \%$. En otras palabras, este resultado indica que más del $50 \%$ de la varianza del constructo se debe a sus indicadores. 
A continuación, se realizó el cálculo de la raíz cuadrada de AVE para testear la validez discriminante de la herramienta, que indica en qué nivel de relación se encuentran dos factores que tratan de medir un mismo constructo. Los valores de la Tabla 4 indican que ningún ítem se repetía en otro de los factores al obtener como resultado valores diferentes de 1 .

Tabla 4

Matriz de correlaciones y raíz cuadrada de AVE.

\begin{tabular}{lllll} 
& ADCM & PPIM & AAM & AAMENF \\
\hline ADCM & 0.919 & & & \\
PPIM & & 0.916 & & \\
AAM & & & 0.820 & 0.937 \\
AAMENF & & & & \\
\hline
\end{tabular}

Posteriormente, se realizó un análisis factorial exploratorio a través de un modelo de ecuaciones estructurales ESEM (Exploratory Structural Equation Modeling) sobre los 13 ítems, utilizando como base el AFE de 4 factores.

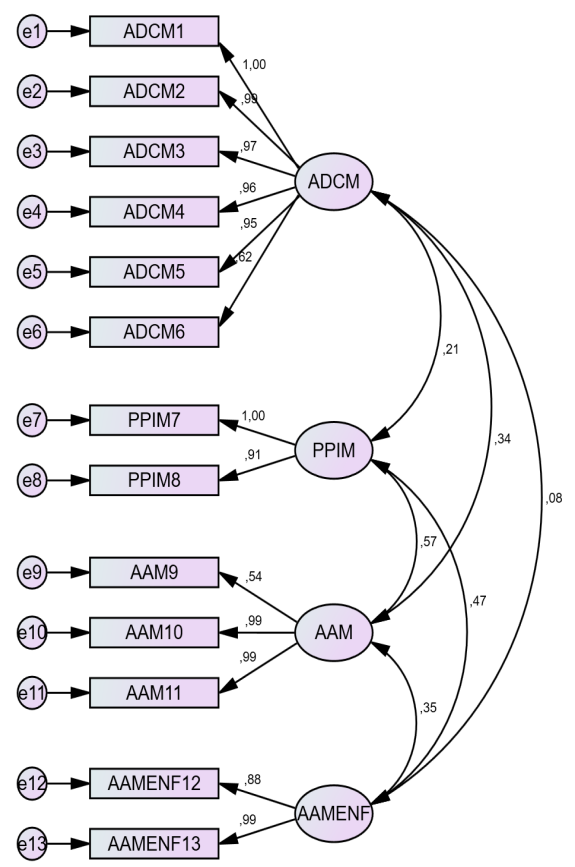

Chi-Square $=498.993, \mathrm{df}=59, \mathrm{P}<0.00$, RMSEA=0.078

Figura 1. Modelo propuesto de ansiedad hacia las matemáticas. 
Los índices de ajuste del modelo de medida ofrecen los siguientes valores: Chi cuadrado $\left(\chi 2_{59}=498.993(\mathrm{p}<.000)\right)$, Comparative Fit Index (CFI) (.982), Incremental Fit Index (IFI) (.982), en ambos casos superior a.90 y Root Mean Square Error of Approximation (RMSEA) (.078), que siendo menor que.08 se acepta la hipótesis nula de que el modelo tiene un ajuste perfecto.

La Figura 1, que corresponde con el modelo resultado, presenta coeficientes de regresión positivos, que indican una relación directa.

Con respecto a los coeficientes de regresión superiores a.70, los alumnos opinan que su ansiedad hacia las matemáticas se relaciona con "la demostración de conocimientos matemáticos", la "preocupación ante la presencia inevitable de las matemáticas", la "ansiedad hacia la acción matemática" y la "ansiedad hacia la actividad matemática en entorno no formal". A excepción de "escuchar cómo otros compañeros resuelven un problema de matemáticas" y "encontrar una página llena de problemas cuando abren el libro", que se relacionan con valores inferiores a.70

\section{Análisis de regresión lineal múltiple}

El segundo de los objetivos planteados en este estudio hace referencia a analizar en qué medida la calificación que obtuvieron el curso pasado en matemáticas está asociada con "Ansiedad hacia la demostración de conocimientos matemáticos", "Preocupación ante la presencia inevitable de las matemáticas", "Ansiedad hacia la acción matemática" y "Ansiedad hacia la actividad matemática en entorno no formal". La variable rendimiento en matemáticas la hemos analizado a través del ítem del instrumento "calificación que obtuviste en matemáticas en el curso pasado".

Para comprobar si la ansiedad hacia las matemáticas predice el rendimiento en matemáticas con dependencia del curso académico, se realizó un análisis de regresión lineal múltiple Stepwise para cada una de las categorías de curso (Tabla 5).

Tabla 5

Modelos de variables predictoras de ansiedad hacia las matemáticas sobre el rendimiento en matemáticas.

\begin{tabular}{cccccccc}
\hline Modelo & R2 & $\begin{array}{c}\text { Error } \\
\text { estándar }\end{array}$ & $\begin{array}{c}\text { Cambio } \\
\text { en R2 }\end{array}$ & $\begin{array}{c}\text { Cambio } \\
\text { en F }\end{array}$ & g12 & Sig. & Durbin-Watson \\
\hline $1^{\text {o }}$ ESO &, 043 & 1,4659 &, 018 & 5,456 & 290 &, 020 & 2,173 \\
$2^{\text {o }}$ ESO &, 046 & 1,5000 &, 022 & 6,774 & 295 &, 010 & 2.194 \\
$4^{\circ}$ ESO & \multirow{2}{*}{148} & 1,4118 &, 018 & 6,379 & 303 &, 012 & 2,206 \\
\hline
\end{tabular}

Podemos decir que los resultados, para $1^{\circ}$ de ESO presentan un modelo que explica un $4,3 \%(\mathrm{p}=, 020)$ de la variable rendimiento en matemáticas, para tres variables: "Me siento nervioso cunado quiero averiguar el cambio en la tienda", "Me pone nervioso hacer operaciones matemáticas" y "Estoy nervioso al recibir las notas finales (del examen) de matemáticas". 
En $2^{\circ}$ de ESO el modelo de tres variables explica un $4,6 \%(p=, 010)$ de la variable dependiente, siendo éstas: "Me pongo nervioso cuando abro el libro de matemáticas y encuentro una página llena de problemas", "Me siento nervioso cuando quiero averiguar el cambio en la tienda" y "Me siento nervioso cuando empiezo a hacer los deberes".

En $3^{\circ}$ de ESO no se encuentra ninguna predicción estadística significativa, pero si aparecen valores cercanos como para la variable "Me siento nervioso al tener que explicar un problema al profesor" $(p=, 124)$.

Para el alumnado de $4^{\circ}$ de ESO el modelo generado consta de cuatro variables que explican un 14,8\% ( $\mathrm{p}=, 012)$ del rendimiento en matemáticas: "Me pongo nervioso cuando abro el libro de matemáticas y encuentro una página llena de problemas", "Me pongo nervioso cuando me doy cuenta de que el próximo curso aun tendré clases de matemáticas", "Me ponen nervioso los exámenes de matemáticas" y "Me pone nervioso hacer operaciones matemáticas".

Para confirmar la validez de los modelos se deben cumplir los supuestos de multicolinealidad perfecta, independencia y homocedasticidad de los residuos y el de linealidad.

La independencia de los residuos se comprueba con la prueba de Durbin-Watson, que cumple en todos los casos por arrojar valores cercanos a 2 (Tabla 5).

El supuesto de multicolinealidad conlleva que las variables de entrada no pueden estar altamente correlacionadas entre sí. Este carácter independiente se comprueba a través del Factor de Inflación de la Varianza (FIV) en la Tabla 6, siendo en todos los casos inferior a 10 por lo que las variables son independientes.

Tabla 6

Coeficientes de los modelos para la variable rendimiento matemático.

\begin{tabular}{|c|c|c|c|c|c|c|c|c|}
\hline & \multirow[t]{2}{*}{ Modelo } & \multicolumn{2}{|c|}{$\begin{array}{l}\text { Coeficientes no } \\
\text { estandarizados }\end{array}$} & \multirow{2}{*}{$\begin{array}{c}\text { Coeficientes } \\
\text { estandarizados } \\
\text { Beta }\end{array}$} & \multirow{2}{*}{$t$} & \multirow{2}{*}{ Sig. } & \multicolumn{2}{|c|}{$\begin{array}{c}\text { Estadísticas de } \\
\text { colinealidad }\end{array}$} \\
\hline & & B & Desv. Error & & & & Tolerancia & VIF \\
\hline \multirow{4}{*}{$1^{\circ} \mathrm{ESO}$} & (Constante) & 5,529 & ,367 & & 15,069 & ,000 & & \\
\hline & $\mathrm{V} 1$ &,- 209 & ,079 &,- 163 & $-2,662$ & ,008 & 871 & 1,149 \\
\hline & $\mathrm{V} 2$ & ,312 & ,095 & ,217 & 3,274 & ,001 & ,742 & 1,347 \\
\hline & V3 &,- 227 & ,097 &,- 148 & $-2,336$ & ,020 & ,811 & 1,233 \\
\hline \multirow{4}{*}{$2^{\circ} \mathrm{ESO}$} & (Constante) & 5,651 & ,240 & & 23,509 & ,000 & & \\
\hline & $\mathrm{V} 1$ &,- 223 & ,086 &,- 148 & $-2,601$ & ,010 & ,984 & 1,016 \\
\hline & $\mathrm{V} 2$ & ,470 & 138 & ,384 & 3,413 & ,001 & ,253 & 3,953 \\
\hline & V3 &,- 404 & 155 &,- 292 & $-2,603$ & ,010 & ,254 & 3,933 \\
\hline
\end{tabular}




\begin{tabular}{|c|c|c|c|c|c|c|c|c|}
\hline & \multirow[t]{2}{*}{ Modelo } & \multicolumn{2}{|c|}{$\begin{array}{l}\text { Coeficientes no } \\
\text { estandarizados }\end{array}$} & \multirow{2}{*}{$\begin{array}{c}\text { Coeficientes } \\
\text { estandarizados } \\
\text { Beta }\end{array}$} & \multirow{2}{*}{$\mathbf{t}$} & \multirow{2}{*}{ Sig. } & \multicolumn{2}{|c|}{$\begin{array}{c}\text { Estadísticas de } \\
\text { colinealidad }\end{array}$} \\
\hline & & B & Desv. Error & & & & Tolerancia & VIF \\
\hline \multirow{5}{*}{$4^{\circ} \mathrm{ESO}$} & (Constante) & 7,490 & ,366 & & 20,493 & ,000 & & \\
\hline & V1 &,- 651 & 172 &,- 444 & $-3,777$ & ,000 & 201 & 4,980 \\
\hline & V2 &,- 520 & 101 &,- 340 & $-5,136$ & ,000 & 633 & 1,580 \\
\hline & V3 &,- 284 & ,083 &,- 188 & $-3,426$ & ,001 & ,918 & 1,090 \\
\hline & V4 & ,443 & 175 & ,323 & 2,526 & 012 & 170 & 5,894 \\
\hline
\end{tabular}

La Figura 2 aporta información sobre el cumplimiento de la homocedasticidad de los residuos y el supuesto de linealidad, ya que la varianza es igual en todos los niveles y no existe ningún patrón no lineal en ninguna de las nubes de puntos.
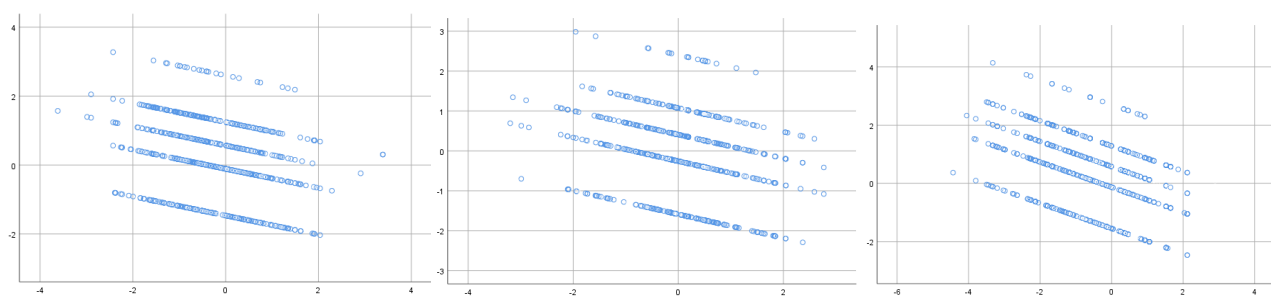

Figura 2. Gráficos de dispersión de valores pronosticados vs residuos tipificados (modelos 1, 2 y 3).

Finalmente, la distribución de los residuos se comprueba a través de la Figura 3, que sigue un patrón muy cercano a la normalidad, en la que los factores se representan en puntos próximos a las líneas.
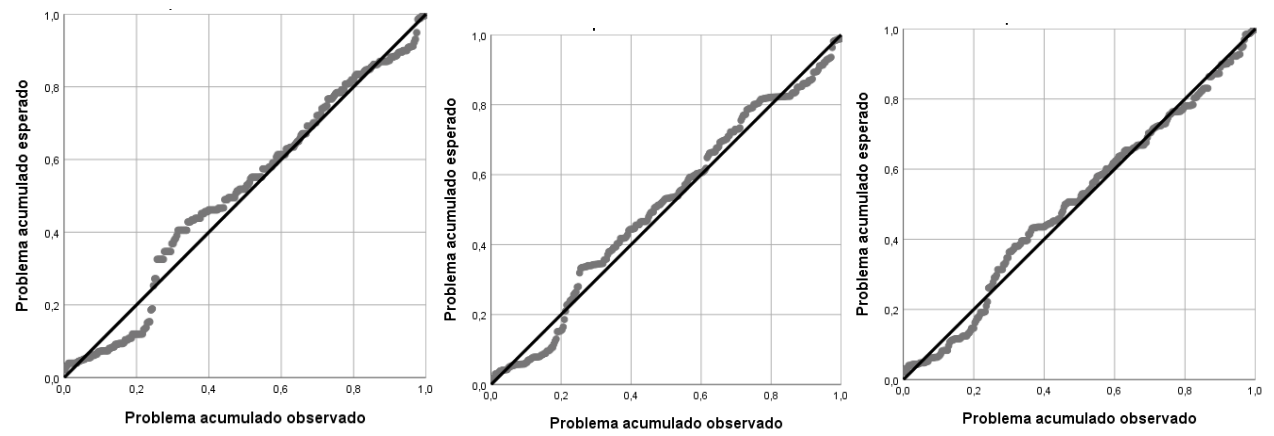

Figura 3. Gráficos P-P normal de regresión de residuo tipificado (modelos 1, 2 y 3).

Comprobados todos los supuestos necesarios, se presentan en la Tabla 7 los resultados correspondientes a los análisis de varianza ANOVA que indican que los 
modelos son buenos para explicar la relación que existe entre las variables de entrada y de salida, ya que el valor de probabilidad es menor de. 05 en los tres casos.

Tabla 7

ANOVA.

\begin{tabular}{llccccc}
\hline \multicolumn{2}{c}{ Modelo } & Suma de cuadrados & gl & Media cuadrática & F & Sig. \\
\hline \multirow{2}{*}{$3^{\text {o }}$ ESO } & Regresión & 34,937 & 3 & 11,646 & 5,419 &, 001 \\
& Residuo & 623,227 & 290 & 2,149 & & \\
& Total & 658,163 & 293 & & & \\
\multirow{2}{*}{$2^{\text {o }}$ ESO } & Regresión & 39,192 & 3 & 13,064 & 5,806 &, 001 \\
& Residuo & 663,805 & 295 & 2,250 & & \\
& Total & 702,997 & 298 & & 14,358 &, 000 \\
& Regresión & 114,478 & 4 & 28,620 & & \\
$4^{\text {o }}$ ESO & Residuo & 603,973 & 303 & 1,993 & & \\
& Total & 718,451 & 307 & & & \\
\hline
\end{tabular}

\section{Discusión y conclusiones}

En este trabajo se ha tratado de comprender la ansiedad hacia las matemáticas en la Enseñanza Secundaria Obligatoria, y poner de relieve los factores que conforman este constructo, a través de evidencias relativas a la fiabilidad y validez de una propuesta de estructura factorial, con una amplia y numerosa muestra, para reducir el error muestral y garantizar su potencia estadística. Los resultados de los análisis realizados permiten confirmar una estructura en cuatro factores que explica un 89,150\% de la ansiedad de los adolescentes hacia las matemáticas, que por orden de mayor a menor varianza son: "Ansiedad hacia la demostración de conocimientos matemáticos", "Preocupación ante la presencia inevitable de las matemáticas", "Ansiedad hacia la acción matemática" y "Ansiedad hacia la actividad matemática en entorno no formal", y que, si bien se aleja de otras propuestas, ofrecen una visión diferente, que con menos variables alcanza un porcentaje de explicación mayor.

Es necesario señalar la presencia de sólo dos ítems en dos factores. No obstante, a pesar de ser un número bajo, los resultados de los análisis confirmatorios avalan su buen funcionamiento.

Los índices de ajuste del modelo de medida indican un ajuste perfecto y presenta coeficientes de regresión positivos, lo que indica que poseen una relación directa.

Así mismo, el sentir de los alumnos es que la ansiedad hacia las matemáticas se relaciona con "la demostración de conocimientos matemáticos", la "preocupación ante la presencia inevitable de las matemáticas", la "ansiedad hacia la acción matemática" y la "ansiedad hacia la actividad matemática en entorno no formal". No es así en el 
caso de "escuchar cómo otros compañeros resuelven un problema de matemáticas" y "encontrar una página llena de problemas cuando abren el libro". Richardson y Suinn, (1972) señalaban que la actividad matemática al usar números y resolver problemas matemáticos produce inquietud tanto en situaciones académicas como en la vida cotidiana.

En cuanto a la relación de los factores con la calificación que obtuvieron el curso pasado en matemáticas, se puede señalar que está asociada con "Ansiedad hacia la demostración de conocimientos matemáticos", "Preocupación ante la presencia inevitable de las matemáticas", "Ansiedad hacia la acción matemática" y "Ansiedad hacia la actividad matemática en entorno no formal". Se confirma que la ansiedad hacia las matemáticas puede influir en el proceso de enseñanza y de aprendizaje. Estas deducciones son compatibles con las halladas en los trabajos de Hannula (2012) en los que explica que el estudiante que padece ansiedad se inquieta profundamente cuando se enfrenta a operaciones numéricas o aritméticas.

También se puede apuntar que tras analizar si la ansiedad hacia las matemáticas predice el rendimiento en matemáticas con dependencia del curso académico, los resultados para $1^{\circ}$ de ESO presentan un modelo con tres variables: "Me siento nervioso cunado quiero averiguar el cambio en la tienda", "Me pone nervioso hacer operaciones matemáticas" y "Estoy nervioso al recibir las notas finales (del examen) de matemáticas". Las respuestas de los alumnos reflejan que su ansiedad hacia las matemáticas se relaciona con "la demostración de conocimientos matemáticos", coincidiendo con lo dicho por Bekdemir (2010), al apuntar que los escolares se ponen nerviosos ante los exámenes y cuando se les llama para explicar un problema o para contestar preguntas.

Por su parte, en $2^{\circ}$ de ESO el modelo, también con tres variables es: "Me pongo nervioso cuando abro el libro de matemáticas y encuentro una página llena de problemas", "Me siento nervioso cuando quiero averiguar el cambio en la tienda" y "Me siento nervioso cuando empiezo a hacer los deberes". Esto es debido a que la realización de las tareas cognitivas se ve alterada (Hannula, 2012).

Por el contrario, en $3^{\text {o }}$ de ESO no se encuentra ninguna predicción estadística significativa, aunque aparecen valores cercanos para la variable "Me siento nervioso al tener que explicar un problema al profesor".

En $4^{\circ}$ de ESO el modelo generado consta de cuatro variables que predicen el rendimiento en matemáticas: "Me pongo nervioso cuando abro el libro de matemáticas y encuentro una página llena de problemas", "Me pongo nervioso cuando me doy cuenta de que el próximo curso aun tendré clases de matemáticas", "Me ponen nervioso los exámenes de matemáticas" y "Me pone nervioso hacer operaciones matemáticas", con un porcentaje de predicción del 14,8\%, muy superior a los resultados en cursos inferiores.

Resaltamos que, aparte de la evidente función y utilidad en la realización del presente estudio, esta información pretende invitar a los docentes, tradicionalmente más atentos a los elementos cognitivos y procedimentales de la instrucción, a considerar los aspectos afectivos y motivacionales con el suficiente grado de importancia y rigor, teniendo en cuenta su constatada influencia tanto en los procesos como en los resultados del aprendizaje. 
Las conclusiones expuestas nos sugieren algunos puntos de reflexión para los investigadores y que consideramos como algunos temas que no han podido ser estudiados, pero que quedan abiertos para futuras investigaciones:

- Comparar la ansiedad hacia las matemáticas en los estudiantes a través de diferentes metodologías.

- Analizar la ansiedad de los profesores hacia las matemáticas.

- Examinar los recuerdos de los profesores sobre la enseñanza de matemáticas cuando eran alumnos.

- Comparar la ansiedad hacia las matemáticas entre alumnos de Primaria y de Secundaria.

\section{Referencias}

Ahmed, W., Minnaert, A., Kuyper, H. \& Werf, G. (2012). Reciprocal relationships between math self-concept and math anxiety. Learning and Individual Differences, 22(3), 385-389.

Ashcraft, M. H. (2002). Math Anxiety: Personal, Educational, and Cognitive Consequences. Current Directions in Psychological Sciences, 11(5), 181-185. Recuperado de http://cdp.sagepub.com/content/11/5/181.short

Asikhia, O. A. y Mohangi, K. (2015). The use of problem-solving training in reducing mathematics anxiety among Nigerian secondary school students. Gender $\mathcal{E}$ Behaviour, 13(1), 6547.

Bazán, J. L. y Aparicio, A. S. (2006). Las actitudes hacia la Matemática- Estadística dentro de un modelo de aprendizaje. Sinéctica. Revista Semestral del Departamento de Educación, 15(28), 1-12. Recuperado de http://revistas.pucp.edu.pe/index.php/ educacion/article/view/2041

Bekdemir, M. (2010). The pre-service teacher's mathematics anxiety related to depth of negative experiences in mathematics classroom while they were students. Educational Studies in Mathematics, 75, 311-328.

Belbase, S. (2013). Images, anxieties and attitudes toward mathematics. International Journal of Education in Mathematics, Science and Technology, 1(4), 230-237.

Blanco, L., Guerrero, E. y Caballero, A. (2013). Cognition and Affect in Mathematics Problem Solving with Prospective Teachers. The Mathematics Enthusiast, 10(1-2), 335-364.

Broc Cavero, M. A. (2006). Motivación y rendimiento académico en alumnos de Educación Secundaria Obligatoria y Bachillerato LOGSE. Revista de Educación, 340, 379414. Recuperado de http://www.ince.mec.es/revistaeducacion/re340/re340_14.pdf

Caballero, A., Guerrero, E. y Blanco, L. J. (2014). Construcción y administración de un instrumento para la evaluación de los afectos hacia las matemáticas. Campo Abierto. Revista de Educación, 33(1), 47-72.

Carrell, S. E., Page, M. E. y West, J. E. (2010). Sex and science: How professor gender perpetuates the gender gap. The Quarterly Journal of Economics, 125, 1101-1114. doi.: 10.1162/qjec.2010.125.3.1101

Casis, M., Rico, N. y Castro, E. (2017). Motivación, autoconfianza y ansiedad como descriptores de la actitud hacia las matemáticas de los futuros profesores de educación básica de Chile. PNA, 11(3), 181-203. 
Chin, W., Peterson, R. A. y Brown, S. P. (2008). Structural equation modeling in marketing: some practical reminders. Journal of Marketing Theory and Practice, 4(16), 287-298. doi.:10.2753/MTP1069-6679160402

Delgado, B.; Inglés, C. J. y García-Fernández, J. M. (2013). La ansiedad social y el autoconcepto en la adolescencia. Revista de Psicodidáctica, 18(1), 179-195.

Eden, C., Heine, A. y Jacobs, A. M. (2013). Mathematics anxiety and its development in the course of formal schooling-a review. Psychology, 4(06), 27-35.

Frenzel, A. C., Pekrun, R. y Goetz, T. (2007). Girls and mathematics- A "hopeless" issue? A control-value approach to gender differences in emotions towards mathematics. European Journal of Psychology of Education, 22(4), 497-514. Recuperado de http://link. springer.com/article/10.1007\%2FBF03173468

Galla, B.M. y Wood, J.J. (2012). Emotional self-efficacy moderates anxiety-related impairments in math performance in elementary school-age youth. Personality and Individual Differences, 52, 118-122.

Gamboa-Araya, R. y Moreira-Mora, T. (2016). Un modelo explicativo de las creencias y actitudes hacia las Matemáticas: Un análisis basado en modelos de ecuaciones estructurales. AIEM, 10, 27-51.

Gómez-Chacón, I. M. (2010). Tendencias actuales en investigación en matemáticas y afecto. En M. M. Moreno, A. Estrada, J. Carrillo y T. A. Sierra, (Eds.). Investigación en Educación Matemática XIV (pp. 121-140). Lleida: SEIEM.

Gómez-Chacón, I. M., Op't Eynde, P. y de Corte, E. (2006). Creencias de los estudiantes de matemáticas. La influencia del contexto de clase. Enseñanza de las Ciencias, 24(3), 309-324. Recuperado de http://www.mat.ucm.es/ imgomezc/cont/docs/12.pdf

González-Montesinos, M. J. y Backhoff, E. (2010). Valoración de un cuestionario de contexto para evaluar sistemas educativos con Modelos de Ecuaciones Estructurales. Revista Electrónica de Investigación y Evaluación Educativa, 2(16). Recuperado de http://www.uv.es/RELIEVE/v16n2/RELIEVEv16n2_1.htm

Gresham, G. (2004). Mathematics Anxiety in elementary students. California Math Council. ComMuniCator, 29(2) 28-29.

Hannula, M. S. (2012). Exploring new dimensions of mathematics-related affect: embodied and social theories. Research in Mathematics Education 14(2), 137-161.

Iriarte, C. y Benavides, M. (2011). Evaluación del programa para superar la ansiedad hacia las matemáticas: PAM. International Journal of Developmental and Educational Psycology, 2(1), 65-74.

Khatoon, T. y Mahmood, S. (2010). Mathematics Anxiety Among Secondary School Students in India and its Relationship to Achievement in Mathematics. European Journal of Social Sciences, 16(1), 75-86

Khezri, H., Lavasania, M. G., Malahmadia, E. y Amania, J. (2010). The role of selfefficacy, task value, and achievement goals in predicting learning approaches and mathematics achievement. Procedia Social and Behavioral Sciences, 5, 942-947. DOI: 10.1016/j.sbspro.2010.07.214.

Loehlin, J. C. (2004). Latent variable models: an introduction to factor, path, and structural

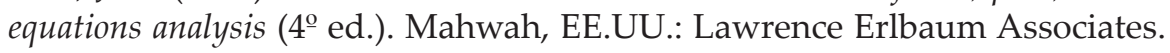

Mato-Vázquez, D. (2006). Diseño y validación de dos cuestionarios para evaluar las actitudes y la ansiedad hacia las Matemáticas en alumnos de educación secundaria 
obligatoria (Tesis doctoral, Universidade da Coruña). Recuperada de http://hdl. handle.net/2183/12688

Mato-Vázquez, D. (2014). Aprender para enseñar Matemáticas en Educación Infantil. Madrid: Pearson.

McLeod, D. B. (1992). Research on affect in mathematics education: A reconceptualization. Handbook of Research on Mathematics Teaching and Learning, 575-596.

Ministerio de Educación, Cultura y Deporte (2012). Datos y Cifras curso escolar 2010/2011. Madrid: MECD.

Ministerio de Educación, Cultura y Deporte (2013). Programa para la evaluación internacional de alumnos de la OCDE (PISA 2012) (Informe Español, Secretaría General Técnica Subdirección General de Información y Publicaciones). Recuperado de http://www.mecd.gob.es/inee/estudios/pisa.html

N.C.T.M. (National Council of Teachers of Mathematics). (2003). Principios y Estándares para la educación matemática. Sevilla: S.A.E.M. "Thales".

Núñez-Peña, M. I., Suárez-Pellicioni, M. y Bono, R. (2013). Effects of math anxiety on student success in higher education. International Journal of Educational Research, $58,36-43$.

OECD (2012). PISA 2009 Technical Report. París: OECD Publishing.

Palacios, A.; Arias, V. y Arias, B. (2014). Las actitudes hacia las matemáticas: construcción y validación de un instrumento para su medida. Revista de Psicodidáctica, 19(1), 67-91.

Pérez-Tyteca, P., Monje, J. y Castro, E. (2013). Afecto y matemáticas. Diseño de una entrevista para acceder a los sentimientos de alumnos adolescentes. Avances de Investigación en Educación Matemática, 4, 65-82.

Richardson, F. C. y Suinn, R. M. (1972). The Mathematics Anxiety Rating Scale: Psychometric Data. Journal of Counseling Psychology, 19, 551-554.

Yara, P. O. (2009). Relationship between teachers' attitude and students' academic achievement in Mathematics in some selected Senior Secondary Schools in Southwestern Nigeria. European Journal of Social Sciences, 11(3), 364-369.

Fecha de recepción: 25 de enero de 2019.

Fecha de revisión: 5 de febrero de 2019.

Fecha de aceptación: 19 de junio de 2019. 
